\title{
Revealing Energy loss and Non-radiative Recombination Pathway in Mixed-ion Perovskite Solar Cells
}

Jialin Dang, Zhi Yang, ${ }^{*}$ Wei Guo, Jinjuan Dou, Hui Wang, Minqiang Wang* Electronic Materials Research Laboratory (EMRL), Key Laboratory of Education Ministry; International Center for Dielectric Research (ICDR); Shaanxi Engineering Research Center of Advanced Energy Materials and Devices, School of Electronic and Information Engineering, Xi'an Jiaotong University, Xi'an, Shaanxi 710049, China.

* Corresponding Author: yangzhi029@xjtu.edu.cn; mqwang@xjtu.edu.cn 
(a)
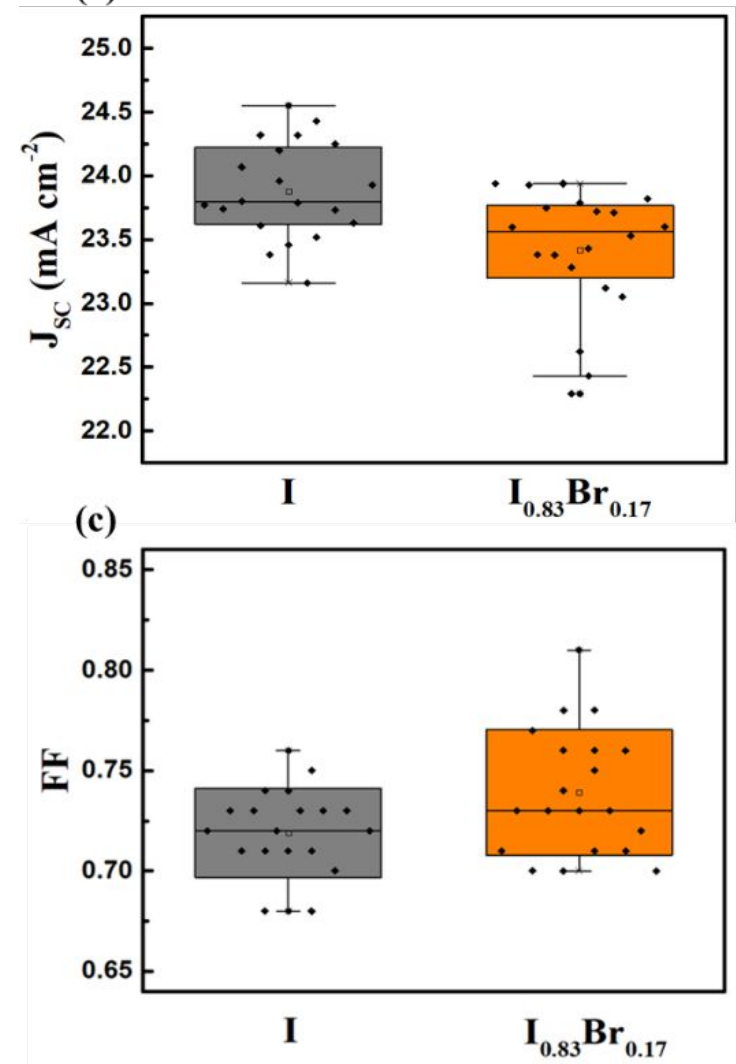

(b)
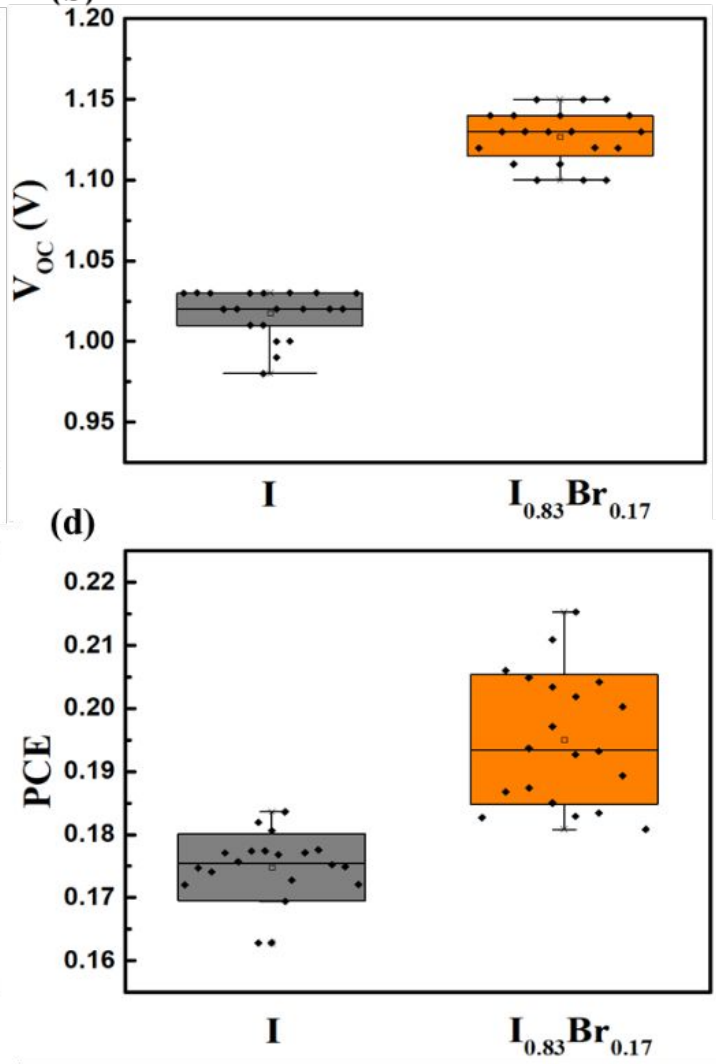

Figure S1. Statistics of (a) $\mathrm{J}_{\mathrm{SC}}$, (b) $\mathrm{V}_{\mathrm{OC}}$, (c) FF, (d) PCE of I and $\mathrm{I}_{0.83} \mathrm{Br}_{0.17}$ devices.

(a)

\begin{tabular}{c|c|c|c|c}
\hline Element & Weight \% & Atomic \% & Net Int. & Error \% \\
\hline $\mathrm{C} \mathrm{K}$ & 8.12 & 34.75 & 51 & 8.41 \\
\hline $\mathrm{N} \mathrm{K}$ & 9.9 & 36.34 & 35.5 & 11.46 \\
\hline $\mathrm{PbM}$ & 27.41 & 6.8 & 326.2 & 3.39 \\
\hline $\mathrm{I} \mathrm{L}$ & 53.48 & 21.68 & 465.6 & 2.91 \\
\hline $\mathrm{CsL}$ & 1.1 & 0.42 & 8.2 & 46.49 \\
\hline
\end{tabular}

(c)

\begin{tabular}{c|c|c|c|c}
\hline Element & Weight \% & Atomic \% & Net Int. & Error \% \\
\hline $\mathrm{C} \mathrm{K}$ & 8.12 & 34.75 & 51 & 8.41 \\
\hline $\mathrm{N} \mathrm{K}$ & 9.9 & 36.34 & 35.5 & 11.46 \\
\hline $\mathrm{BrL}$ & 8.5 & 5.15 & 97.3 & 8.97 \\
\hline $\mathrm{PbM}$ & 27.41 & 6.8 & 326.2 & 3.39 \\
\hline $\mathrm{I} \mathrm{L}$ & 53.48 & 21.68 & 465.6 & 2.91 \\
\hline $\mathrm{CsL}$ & 1.1 & 0.42 & 8.2 & 46.49 \\
\hline
\end{tabular}

Cs:Pb:I:Br= 0.06:1:2.76:0.83

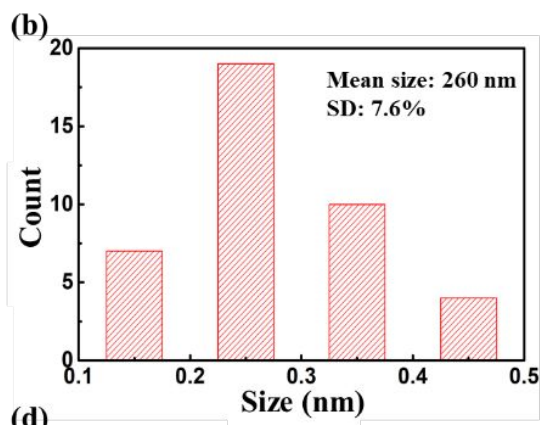

(d)

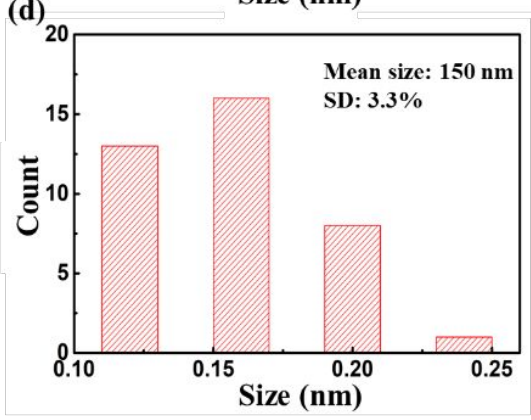

Figure S2. Energy spectrum scanning results of (a) I and (c) $\mathrm{I}_{0.83} \mathrm{Br}_{0.17}$ films, and grain size statistics of (b) I and (d) $\mathrm{I}_{0.83} \mathrm{Br}_{0.17}$ films. 


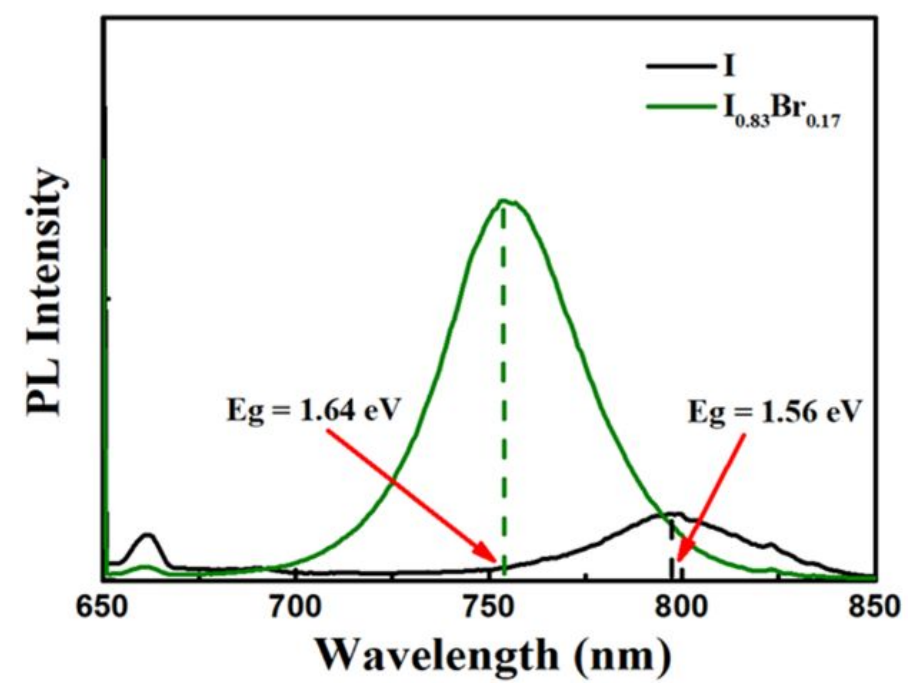

Figure S3. PL spectra of I and $\mathrm{I}_{0.83} \mathrm{Br}_{0.17}$ films deposited on the $\mathrm{SnO}_{2} / \mathrm{ITO}$ substrate.

(a)
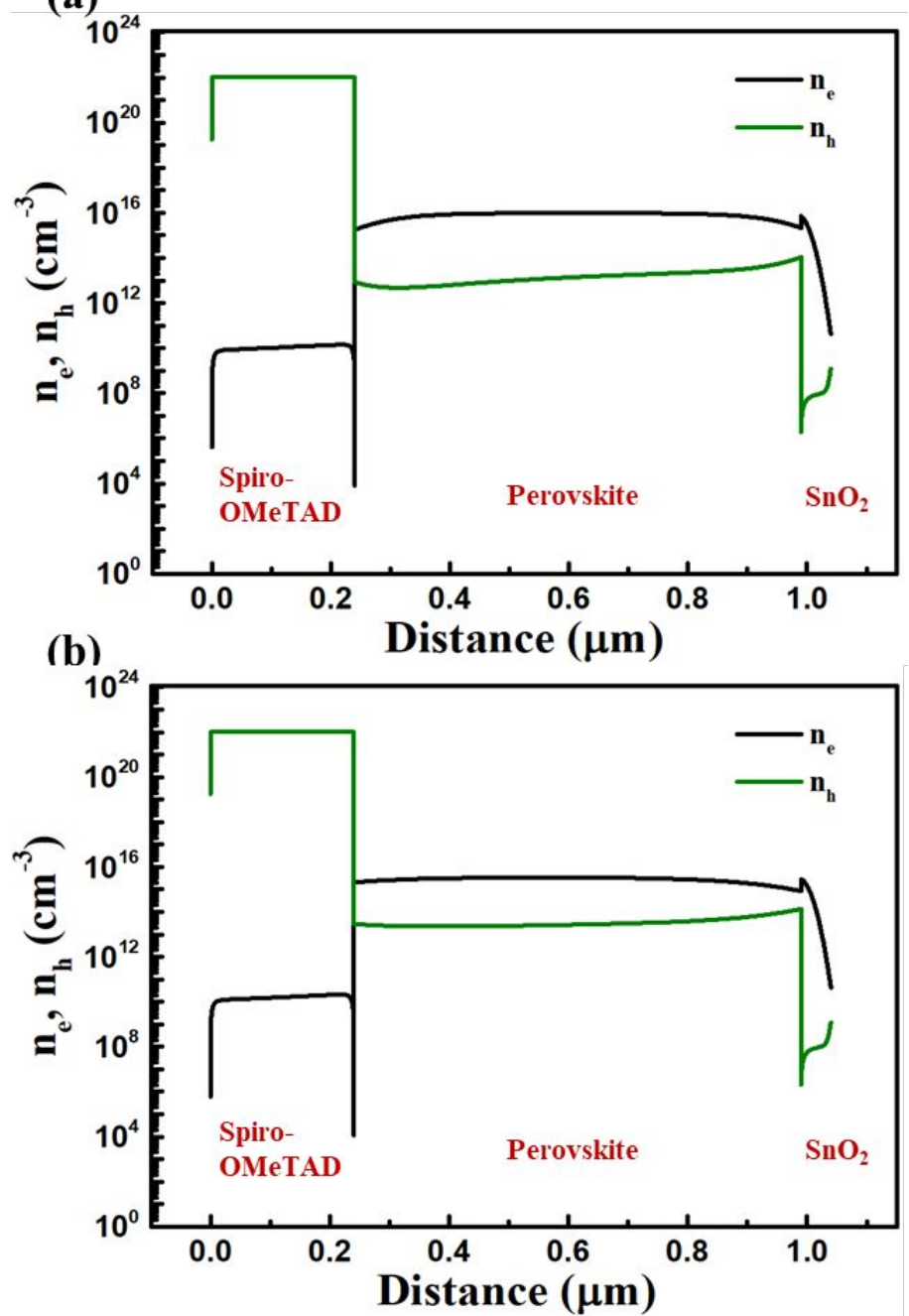

Figure S4. Distribution of the carrier concentration at different positions of (a) I and (b) $\mathrm{I}_{0.83} \mathrm{Br}_{0.17}$ device applied at $\mathrm{V}_{\mathrm{OC}}$ and illuminated under 1 sun. 

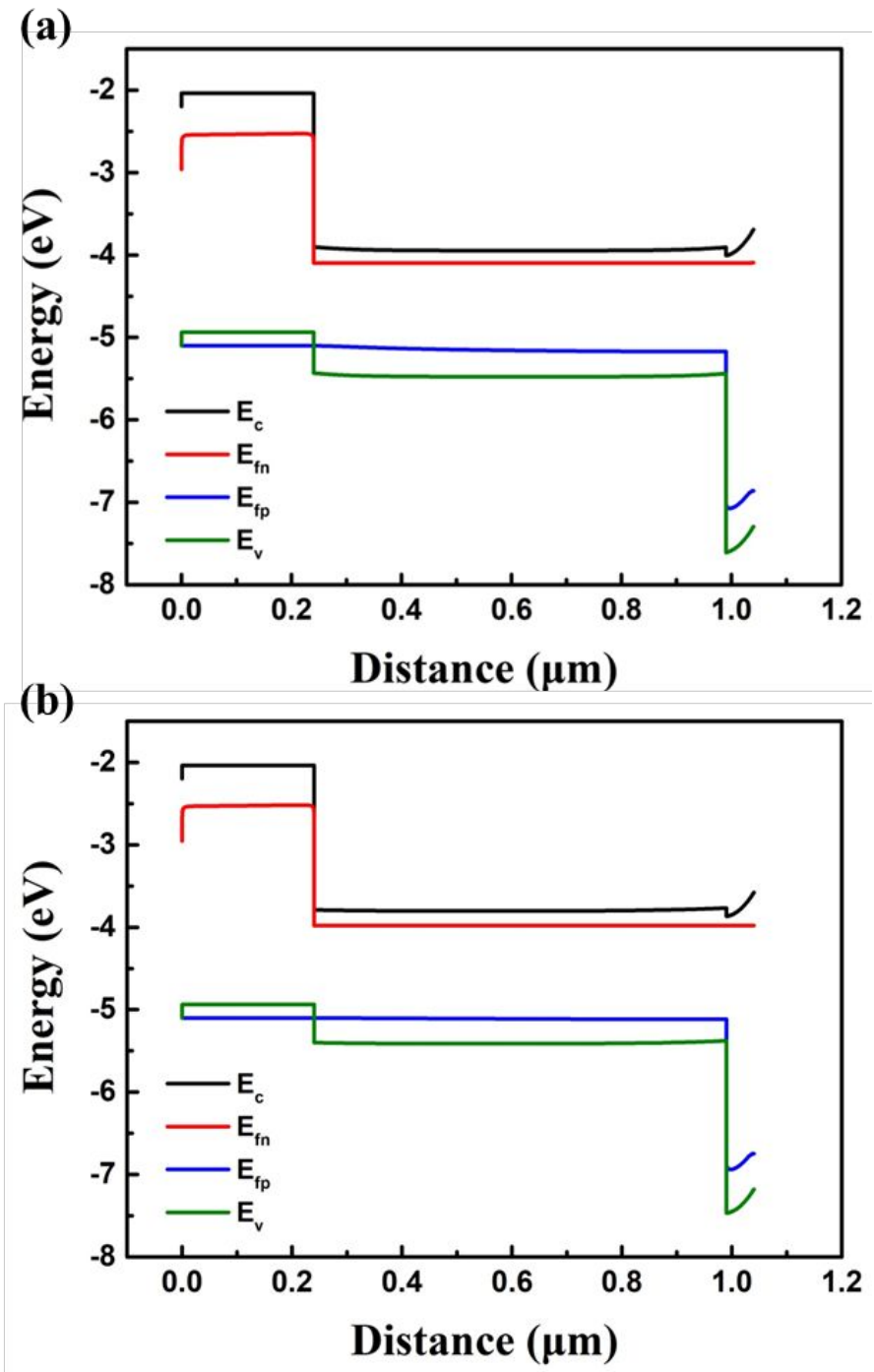

Figure S5. Energy band diagrams of (a) I and (b) $\mathrm{I}_{0.83} \mathrm{Br}_{0.17}$ device applied at $\mathrm{V}_{\mathrm{OC}}$ and illuminated under 1 sun. 


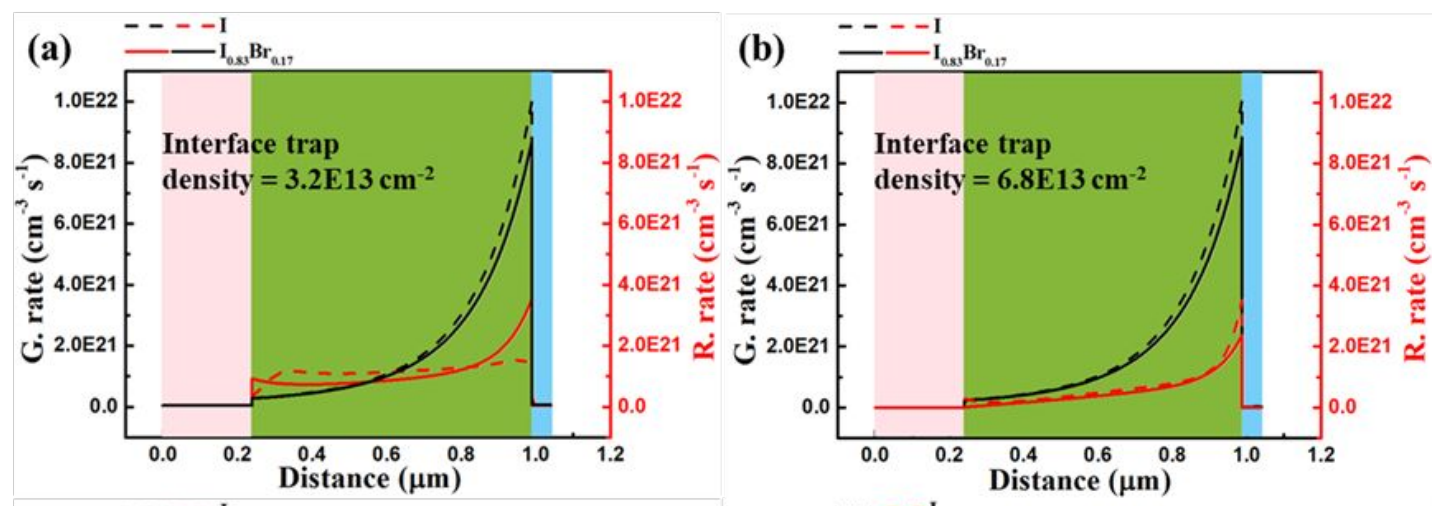

(b) $\stackrel{---1}{-1} \mathrm{I}_{0,3} \mathrm{Br}_{0,1}$

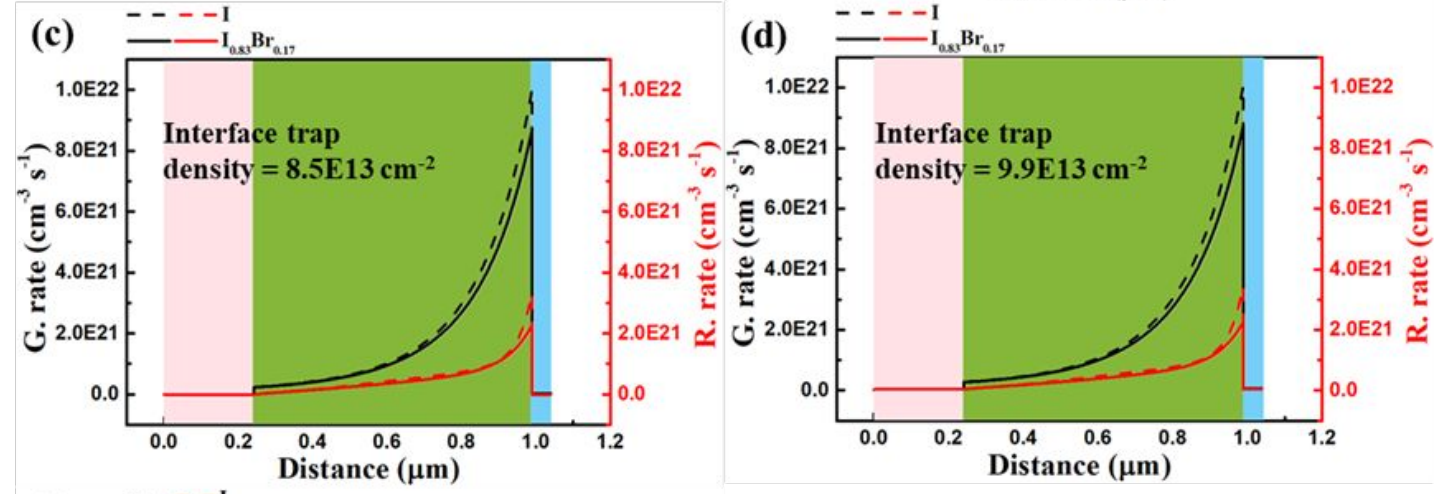

(d) $\quad----1$

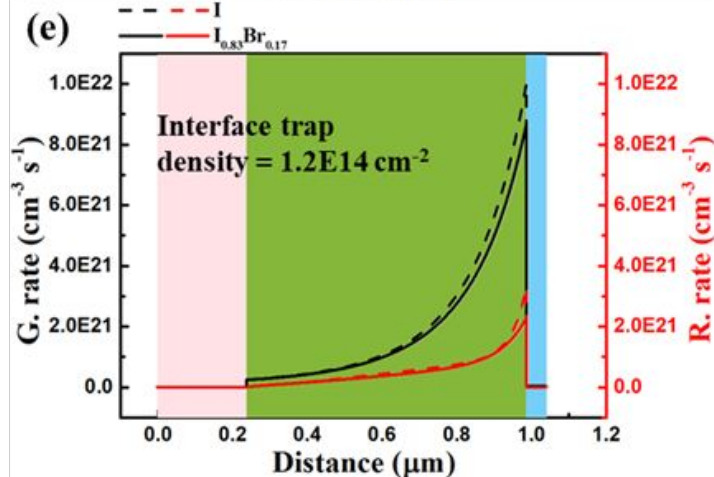

Figure S6. Distribution of generation rate and recombination rate of two devices with different interface trap densities applied at $\mathrm{V}_{\mathrm{OC}}$ and illuminated under 1 sun. The generation rate distribution of the two devices does not change with the increase of trap density under constant light illumination, and the recombination rate changes significantly at the perovskite/HTL interface. 
(a)

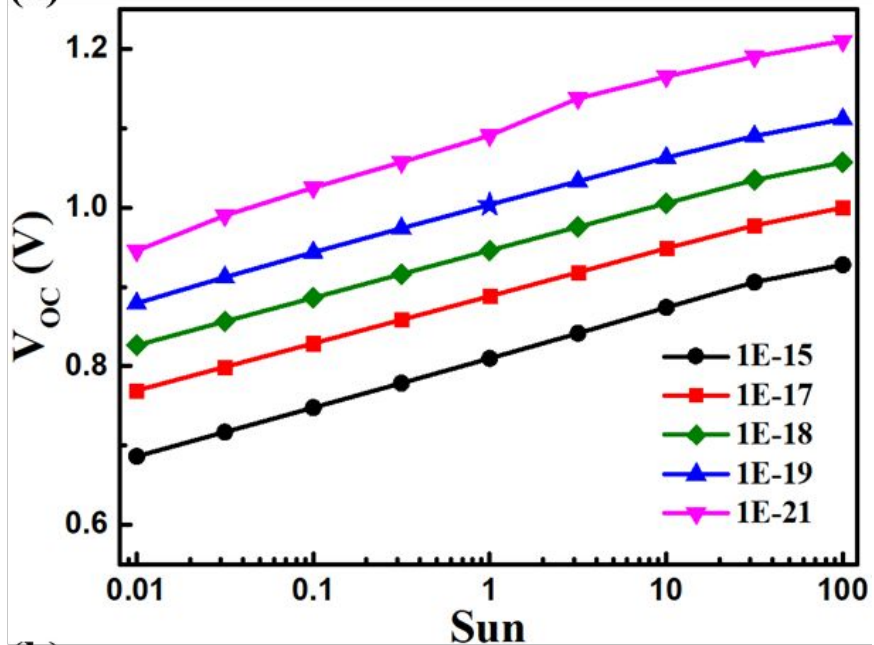

(b)

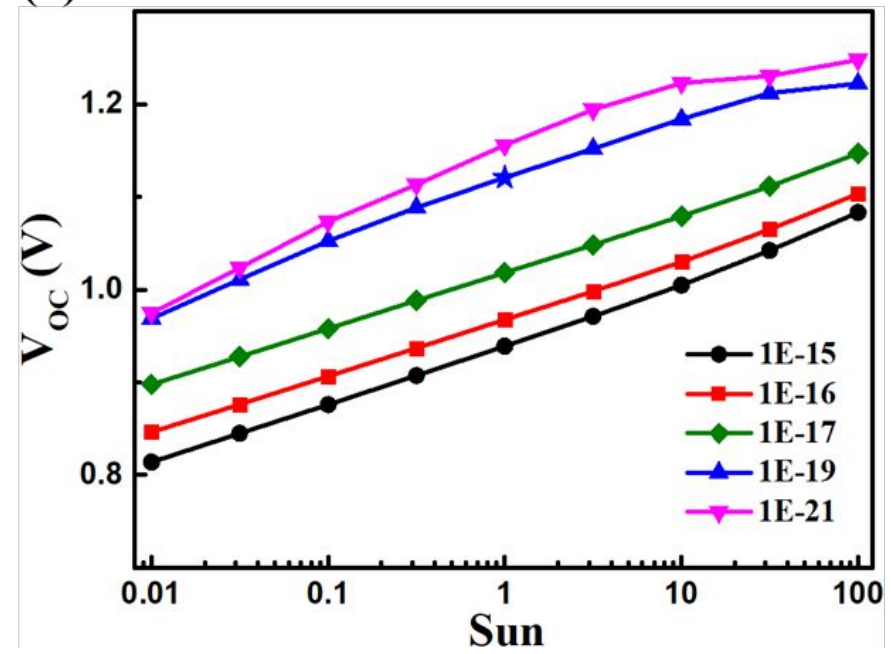

Figure S7. Influence of the carrier capture cross section at the perovskite/HTL interface on the $\mathrm{V}_{\mathrm{OC}}$ of (a) I and (b) $\mathrm{I}_{0.83} \mathrm{Br}_{0.17}$ devices under different light intensities.

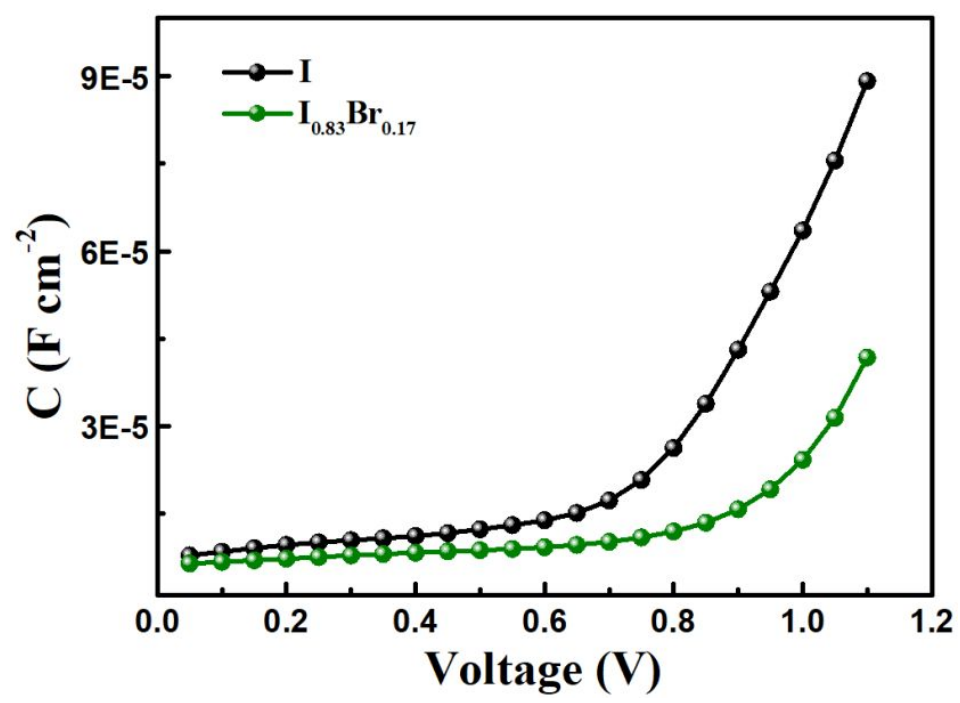

Figure S8. Capacitance-voltage plot extracted from the Nyquist spectra. 
Table S1. Basic parameters of n-i-p MHP solar cells used in SCAPS simulation.

\begin{tabular}{|c|c|c|c|}
\hline Parameters & $\mathrm{SnO}_{2}$ & MHP & $\begin{array}{c}\text { Spiro- } \\
\text { OMeTAD }\end{array}$ \\
\hline Thickness (nm) & 50 & 750 & 240 \\
\hline Bandgap (eV) & 3.6 & $1.61 / 1.53$ & 2.9 \\
\hline Electron affinity $(\mathrm{eV})$ & 4 & 3.9 & 2.2 \\
\hline Dielectric permittivity (relative) & 9 & 22 & 3 \\
\hline $\mathrm{CB}$ effective density of states $\left(\mathrm{cm}^{-3}\right)$ & $2.2 \times 10^{17}$ & $3.1 \times 10^{18}$ & $2.5 \times 10^{18}$ \\
\hline VB effective density of states $\left(\mathrm{cm}^{-3}\right)$ & $2.2 \times 10^{16}$ & $3.1 \times 10^{18}$ & $1.8 \times 10^{19}$ \\
\hline Electron thermal velocity $(\mathrm{cm} / \mathrm{s})$ & $1.00 \times 10^{7}$ & $1.00 \times 10^{7}$ & $1.00 \times 10^{7}$ \\
\hline Hole thermal velocity $(\mathrm{cm} / \mathrm{s})$ & $1.00 \times 10^{7}$ & $1.00 \times 10^{7}$ & $1.00 \times 10^{7}$ \\
\hline Electron mobility $\left(\mathrm{cm}^{2} / \mathrm{Vs}\right)$ & 20 & 10 & $10^{-4}$ \\
\hline Hole mobility $\left(\mathrm{cm}^{2} / \mathrm{Vs}\right)$ & 10 & 10 & $10^{-4}$ \\
\hline $\begin{array}{l}\text { Shallow uniform acceptor density } \mathrm{N}_{\mathrm{A}} \\
\qquad\left(\mathrm{cm}^{-3}\right)\end{array}$ & 0 & 0 & $10^{22}$ \\
\hline $\begin{array}{l}\text { Shallow uniform donor density } \mathrm{N}_{\mathrm{D}} \\
\qquad\left(\mathrm{cm}^{-3}\right)\end{array}$ & $10^{17}$ & $3.5 \times 10^{15} / 9.8 \times 10^{15}$ & 0 \\
\hline Energy distribution (eV) & 0.60 above $\mathrm{E}_{\mathrm{V}}$ & 0.30 below $\mathrm{E}_{\mathrm{C}}$ & 0.60 above $E_{V}$ \\
\hline Total defect density $\left(\mathrm{cm}^{-3}\right)$ & $1.00 \times 10^{15}$ & $3.1 \times 10^{15 / 3.5 \times 10^{15}}$ & $1.00 \times 10^{15}$ \\
\hline
\end{tabular}

Table S2. Parameters of bulk and interface defects in MHPs used in SCAPS simulation.

\begin{tabular}{ccccc}
\hline Parameters & 1.53-MHP & $\begin{array}{c}1.53- \\
\text { MHP/Spiro } \\
\text { interface }\end{array}$ & $\begin{array}{c}1.61-\mathrm{MHP} \\
\text { Defect type }\end{array}$ & $\begin{array}{c}\text { MHP/Spiro } \\
\text { interface }\end{array}$ \\
\hline Single & Single & Single & Single \\
donor & donor & donor & donor \\
Capture cross section electrons $\left(\mathrm{cm}^{2}\right)$ & $1.00 \times 10^{-15}$ & $1.00 \times 10^{-19}$ & $1.00 \times 10^{-15}$ & $1.00 \times 10^{-19}$ \\
Capture cross section holes $\left(\mathrm{cm}^{2}\right)$ & $1.00 \times 10^{-15}$ & $1.00 \times 10^{-19}$ & $1.00 \times 10^{-15}$ & $1.00 \times 10^{-19}$ \\
Energetic distribution & Single & Single & Single & Single \\
$\mathrm{E}_{\mathrm{t}}$ with respect to the lowest $\mathrm{E}_{\mathrm{c}}(\mathrm{eV})$ & 0.3 & 0.6 & 0.3 & 0.6 \\
Total density $\left(\mathrm{cm}^{-2}\right)$ & $3.5 \times 10^{15}$ & $6.8 \times 10^{13}$ & $3.1 \times 10^{15}$ & $3.2 \times 10^{13}$ \\
Carrier lifetime $(\mathrm{ns})$ & 29 & - & 32 & - \\
& & & & - \\
Diffusion length $(\mathrm{nm})$ & 860 & & & - \\
& & & &
\end{tabular}

